\title{
A Hyper-Block Self-Consistent Approach to Nonlinear Schrodinger Equations: Breeding, Metamorphosis and Killing of Hofstadter Butterflies
}

Mehdi solaimani ${ }^{1}$

${ }^{1}$ Qom University of Technology

May 5, 2020

\begin{abstract}
Nonlinear Schrödinger equations play essential roles in different physics and engineering fields. In this paper, a hyper-block finitedifference self-consistent method (HFDSCF) is employed to solve this stationary nonlinear eigenvalue equation and demonstrated its accuracy. By comparing the results with the Sinc self-consistent (SSCF) method and exact available results, we show that the HFDSCF method gives quantum states with high accuracy and can even solve the strongly nonlinear Schrodinger equations. Then, by applying our method to Hofstadter butterfly problem, we describe the breeding, metamorphosis and killing of these butterflies by using nonlinear interactions as well as two constant length multi-well and sinusoidal potentials.
\end{abstract}

\section{Hosted file}

manuscript.pdf available at https://authorea.com/users/298936/articles/428309-a-hyper-blockself-consistent-approach-to-nonlinear-schrodinger-equations-breeding-metamorphosis-andkilling-of-hofstadter-butterflies 